\title{
Modeling the Plasma Flow in the Inner Heliosheath with a Spatially Varying Compression Ratio
}

\author{
G. Nicolaou ${ }^{1}$ and G. Livadiotis ${ }^{2}$ \\ ${ }^{1}$ Swedish Institute of Space Physics, Kiruna, Sweden \\ ${ }^{2}$ Southwest Research Institute, San Antonio, Texas, USA \\ Received 2016 December 27; revised 2017 February 7; accepted 2017 February 18; published 2017 March 17
}

\begin{abstract}
We examine a semi-analytical non-magnetic model of the termination shock location previously developed by Exarhos \& Moussas. In their study, the plasma flow beyond the shock is considered incompressible and irrotational, thus the flow potential is analytically derived from the Laplace equation. Here we examine the characteristics of the downstream flow in the heliosheath in order to resolve several inconsistencies existing in the Exarhos \& Moussas model. In particular, the model is modified in order to be consistent with the RankineHugoniot jump conditions and the geometry of the termination shock. It is shown that a shock compression ratio varying along the latitude can lead to physically correct results. We describe the new model and present several simplified examples for a nearly spherical, strong termination shock. Under those simplifications, the upstream plasma is nearly adiabatic for large ( 100 AU) heliosheath thickness.
\end{abstract}

Key words: plasmas - shock waves - solar wind - Sun: heliosphere

\section{Introduction}

Parker (1958) introduced the theory of the solar wind acceleration. Solar wind is accelerated in the solar corona and flows radially outward with supersonic speed. At the termination shock, the plasma is slowed down, heated, and compressed. The shocked plasma is then flowing in the heliosheath and eventually turns in the direction of the interstellar flow. The boundary between the heliosheath plasma and the interstellar medium is the heliopause.

Parker (1961) presented three different analytical models of stellar wind flow. The simplest model describes the radial flow of stellar wind from a star that is at rest in respect to the nonmagnetized, uniform local interstellar medium (LISM). $\mathrm{He}$ then described the case of an expanding stellar wind from a star that is moving with respect to the non-magnetized medium. In this case, a two-term flow potential was introduced to describe the flow beyond the termination shock, which is considered incompressible and irrotational. Finally, Parker discussed the case of a static medium with significant magnetic field. The scalar flow potential was solved for the cases of minimum and maximum stagnation pressure assuming a strong termination shock (see Figure 3 of that paper, where the outer boundary of the shocked stellar wind is illustrated for several values of the stagnation pressure).

Nerney \& Suess (1995) analytically solved the Laplace equation and derived the potential that describes the solar wind flow beyond the termination shock. They defined the termination shock location by applying pressure balance between the upstream solar wind and the interstellar medium. In their model, a specific speed variation as a function of the latitude (from upwind to downwind flow) was imposed. In this way, they were able to predict, among others, the polar elongation of the termination shock.

Barnes (1998) estimated the geometry of the termination shock by applying the analysis of Parker (1961) and using solar wind parameters that vary with latitude. Following Barnes (1998), Exarhos \& Moussas (2000) developed a semi-analytical model that predicts the heliocentric distance of the heliospheric termination shock as a function of latitude using Ulysses spacecraft observations for the upstream conditions. They combined the Rankine-Hugoniot jump conditions for an oblique strong shock and the flow potential beyond the shock in order to estimate its location as a function of latitude. In their model, the heliosheath thickness in the standoff direction is a free parameter and affects the flow potential solution as well as the shape and the location of the termination shock. While the model allows the estimation of the polar and the caudal elongation using real plasma observations for the upstream conditions, it leads to results that are inconsistent with the shock geometry and jump conditions. Those inconsistencies and their resolution are discussed in this paper.

More recently, Senanayake \& Florinski (2013) developed an analytical model of the termination shock and the heliosheath flow in order to study how the termination shock geometry affects the acceleration of the anomalous cosmic rays. In their study, the flow in the heliosheath is expressed as the curl of a Stokes' stream function that holds the incompressibility condition. They examine a model of an elongated termination shock with a slightly varying compression ratio.

Besides the theoretical models, several numerical gas dynamic and MHD models of the solar wind and LISM interaction have been developed (for details, see Zank 1999; Pogorelov 2016, and references therein). Gas dynamic simulations assume subsonic LISM and a strong termination shock and predict the heliosheath flow, which is qualitatively similar to the heliosheath flow as derived from analytical models. In the case of supersonic LISM, the termination shock is a bullet shaped strong shock and a bow shock is formed in order to divert the LISM flow about the heliosphere (e.g., Baranov \& Malama 1993; Steinolfson 1994; Steinolfson et al. 1994; Pauls et al. 1995; Steinolfson \& Gurnett 1995; Wang \& Belcher 1998). Lipatov et al. (1998) used Boltzmann simulations to study for the first time the characteristics of the neutral hydrogen distribution function at different locations within the heliosphere. Analytic MHD models and 3D MHD simulations have been developed in order to study the interaction of the solar wind and a magnetized LISM for 
different plasma properties and magnetic field orientation (Zank 1999 and references therein).

While sophisticated numerical models and simulations can describe the structures of the heliosphere, analytical and semianalytical models can be useful to represent the basic features under several assumptions and simplifications. In addition to that, calculations with such models can be significantly faster. Lastly, the development of analytical or semi-analytical models is often easy and their computational cost is low. In order to estimate the termination shock location and shape with analytical/semi-analytical models, for specific upstream and interstellar parameters, we need to find a flow potential that is consistent with the jump conditions on the oblique termination shock.

In this study, we examine the general concept and principals originally developed in Exarhos \& Moussas (2000), while we modify the model to obtain reasonable streamlines in the inner heliosheath. We introduce a spatially variant shock compression ratio that ensures, for the consistency of the model, which corresponds to a spatially variant polytropic index (e.g., Livadiotis 2015). We use the new model to present simplified examples of the termination shock and heliopause distance, while we explain how it can be used for more complicated shock geometries and upstream plasma conditions.

The paper is organized as follows. In Section 2, we describe the model of Exarhos \& Moussas (2000) and in Section 3 we discuss its inconsistency for a fixed compression ratio. We then introduce modifications and improvements using a variable compression ratio, while Section 4 presents few simplified results of the new, modified model. A summary of the conclusions and a discussion of the developments and results are placed in Section 5 .

\section{The Exarhos \& Moussas Semi-analytical Non-magnetic Model of the Termination Shock Location}

In this section, we present the non-magnetic model by Exarhos \& Moussas (2000) for the plasma flow upstream and downstream the termination shock. The transition from supersonic (upstream) to subsonic (downstream) plasma flow is described by the hydrodynamic Rankine-Hugoniot jump conditions on the termination shock. We then show how the model calculates the termination shock distance by combining the jump conditions and the equations of the flow and how it may lead to non-physical results when assuming a fixed shock compression ratio. Finally, it is shown that a spatially varying compression ratio can lead to a self-consistent model.

\subsection{Supersonic Solar Wind Flow}

The flow upstream of the termination shock is supersonic, radial, and spherically symmetric (Parker 1958). The solar wind speed at $\sim 1 \mathrm{AU}$ is $\sim 400 \mathrm{~km} \mathrm{~s}^{-1}$. For simplicity, in this study, we assume that the solar wind speed does not change with radial distance (see also the discussion by Exarhos \& Moussas 1999a, 1999b). The conservation of mass implies that the density of the plasma upstream of the shock is proportional to the inverse square of the heliocentric distance. If we know the density of the plasma $\rho_{\mathrm{o}}$ at a heliocentric distance $r_{0}$, then we can calculate the upstream density $\rho_{1}$ at the termination

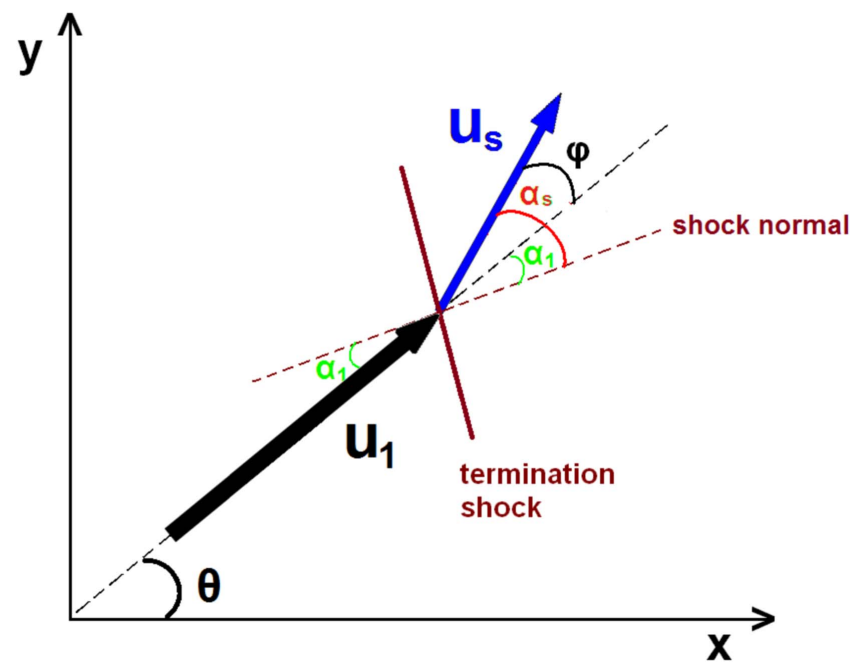

Figure 1. Representation of $\alpha_{1}, \alpha_{s}$ and $\varphi=\alpha_{s}-\alpha_{1}$ angles. The red solid line represents the termination shock surface, while the dashed red line represents the shock normal. The plasma downstream flow vector is represented with the blue arrow.

shock distance $r_{\mathrm{s}}$ (of the same streamline),

$$
\rho_{1}=\rho_{0}\left(\frac{r_{0}}{r_{s}}\right)^{2} .
$$

\subsection{Rankine-Hugoniot Jump Conditions}

At the termination shock, the supersonic solar wind plasma becomes subsonic and then flows downstream of the termination shock. The Rankine-Hugoniot equations (Rankine 1870; Hugoniot 1887; Hugoniot 1889) describe the relationship between the plasma properties on both sides of the shock. The hydrodynamic Rankine-Hugoniot jump conditions at an oblique and strong, infinite Mach number shock (the ratio of upstream thermal to dynamic ram pressure is approximately 0):

$$
\begin{gathered}
\rho_{s}=R \rho_{1}, \\
u_{s} \sin \alpha_{s}=u_{1} \sin \alpha_{1}, \\
u_{s} \cos \alpha_{s}=R^{-1} u_{1} \cos \alpha_{1}, \\
P_{s}=\rho_{1} u_{1}^{2} \cos ^{2} \alpha_{1}\left(1-R^{-1}\right),
\end{gathered}
$$

where $\rho, u$, and $P$ are the plasma density, speed (at the shock's reference frame), and pressure, respectively. The angle between the flow and the front shock normal is denoted by $\alpha$ (Figure 1). Subscript " 1 " denotes parameters upstream of the shock and " $s$ " denotes the parameters downstream of the shock. The compression ratio for the strong shock is

$$
R=\frac{\gamma+1}{\gamma-1}
$$

where $\gamma$ is the polytropic index of the plasma. We note that the last equation is valid only if the polytropic index $\gamma$ of the upstream and the downstream region of the strong shock is the same (for more details, see Livadiotis 2015). In a different case, the above equations are more complicated. Nevertheless, in this study, we keep the strong shock approximation as a first attempt to demonstrate the inconsistencies that can arise in simplified semi-analytical models and provide a simple theoretical approach to a self-consistent model. 


\subsection{The Termination Shock Location}

Exarhos \& Moussas (2000) combined the Rankine-Hugoniot jump conditions, the Bernoulli's integral, and the expressions for the supersonic flow, to end up with the expression below that predicts the location of the termination shock for several latitudes:

$$
\left(\frac{r_{s}}{r_{0}}\right)^{2}=\frac{\rho_{0}}{P_{\infty}+\frac{1}{2} \rho_{\infty} u_{\infty}^{2}} \cdot \frac{u_{1}^{2}+\frac{1}{2} u_{s}^{2}(R-1)}{1+R^{-1}},
$$

where $P_{\infty}, \rho_{\infty}$ and $u_{\infty}$ are the thermal pressure, mass density, and the speed of the LISM, respectively, and $r_{s}$ is the heliocentric distance of the termination shock. We then need the expression of the $u_{s}$ speed as a function of the heliocentric distance to numerically solve Equation (3). Note that Equation (3) can be solved for all latitudes.

\subsection{Subsonic Plasma Flow in the Heliosheath}

In general, the polytropic index of the plasma in the upstream region differs from the polytropic index downstream (e.g., Parker 1961; Livadiotis 2015). For example, while the upstream solar wind has a typical polytropic index around the adiabatic value of $\gamma \sim 5 / 3$ (e.g., Nicolaou et al. 2014; Livadiotis \& Desai 2016), for the downstream plasma in the inner heliosheath, there is no such evidence; on the contrary, there are cases where the measured $\gamma$ is quite different, i.e., near the isobaric value, $\gamma \sim 0$ (e.g., Livadiotis \& McComas 2012, 2013). Following previous studies, we consider the same upstream and downstream polytropic index for the shock transition. However, it can be shown that under some conditions (LISM properties and upstream/downstream polytropic indices) the general flow in the heliosheath can be well approximated as incompressible and irrotational (e.g., Parker 1961; Barnes 1998; Suess \& Nerney 1990; Exarhos \& Moussas 2000). Under this approximation, the velocity in the heliosheath can be given from the scalar potential $\Phi$, which is a solution of the Laplace equation:

$$
\nabla^{2} \Phi=0 .
$$

We set a heliocentric coordinate system where the $x$-axis is toward the Sun's motion relative to the local medium (toward the heliosphere nose) and $y$-axis is toward the north. Assuming symmetry by the $x$-axis, the general solution for $\Phi$ is written as

$$
\Phi=\sum_{n=0}^{\infty}\left(A_{n} r^{n}+B_{n} r^{-(n+1)}\right) P_{n}(\cos \theta),
$$

where $r$ is the heliocentric distance, $P_{\mathrm{n}}$ is the Legendre polynomials, and $\theta$ is the polar angle measured in the $x-y$ plane (latitude). For $\theta=0^{\circ}$, the flow is anti- parallel to the LISM flow relative to the Sun (upwind direction) and for $\theta=90^{\circ}$ is toward the $y$-axis (also, see Figures 1 and 2). Keeping terms of the order of $r^{-2}$ and following the same notation as Exarhos \& Moussas (2000), the general 2D solution for the heliosheath flow potential is

$$
\Phi=A_{0}+\frac{B_{0}}{r}+B r \cos \theta+\frac{\Delta}{r^{2}} \cos \theta,
$$

and the components of the velocity are

$$
u_{r}=-\frac{\partial \Phi}{\partial r}=\frac{B_{0}}{r^{2}}-B \cos \theta+\frac{2 \Delta}{r^{3}} \cos \theta,
$$

$$
u_{\theta}=-\frac{1}{r} \frac{\partial \Phi}{\partial \theta}=B \sin \theta+\frac{\Delta}{r^{3}} \sin \theta .
$$

In the above equations, $A_{0}, B_{0}, B$, and $\Delta$ are constants, determined from the boundary conditions

$$
\begin{gathered}
\boldsymbol{u}(r \rightarrow \infty)=-u_{\infty} \boldsymbol{x}, \\
u\left(r=r_{h}, \theta=0^{\circ}\right)=u_{h 0}=0, \\
u\left(r=r_{s}, \theta=0^{\circ}\right)=u_{s 0}=R^{-1} u_{1},
\end{gathered}
$$

where $r_{h}$ is the heliopause radial distance from the Sun. Hence, the involved constants are given by

$$
\begin{gathered}
B=u_{\infty}, \\
B_{0}=\left(B-\frac{2 \Delta}{r_{h 0}{ }^{3}}\right) \cdot r_{h 0^{2}}, \\
\Delta=\frac{\left(R^{-1} u_{1}+u_{\infty}\right) r_{s 0}{ }^{2}-u_{\infty} r_{h 0}{ }^{2}}{2\left(r_{s 0}{ }^{-1}-r_{h 0^{-1}}\right)},
\end{gathered}
$$

where $r_{s 0}$ and $r_{h 0}$ are the termination shock and the heliopause heliocentric distance for $\theta=0^{\circ} ; r_{s}$ is determined from Equation (3) under condition (11), and $r_{h 0}$ is a free parameter of the model.

\section{Inconsistencies of the Exarhos \& Moussas Model}

\subsection{General Description}

Using Equation (3), we estimate the termination shock location for latitude $\theta=0^{\circ}$ (along the $x$-axis). We use the LISM parameters as used in Pauls \& Zank (1996), which are $u_{\infty}=26 \mathrm{~km} \mathrm{~s}^{-1}$ (in the $-x$ direction), $P_{\infty}=1 \times 10^{-13} \mathrm{~Pa}$ (which also accounts for the magnetic pressure), $n_{\infty}=0.1 \mathrm{~cm}^{-3}$. Typical parameter values of the supersonic upstream solar wind plasma are $u_{1}=400 \mathrm{~km} \mathrm{~s}^{-1}$ and $n_{0}=5 \mathrm{~cm}^{-3}$ at $r_{0}=1 \mathrm{AU}$. Considering a constant compression ratio of the shock $R(\theta=0)=4$, which is the maximum limit of the compression ratio for an adiabatic strong shock Equation (3) under condition (11) gives

$$
r_{s 0}=r_{0} u_{1} \sqrt{\frac{7 \rho_{0}}{8\left(P_{\infty}+\frac{1}{2} \rho_{\infty} u_{\infty}^{2}\right)}} \approx 86 \mathrm{AU}
$$

In order to solve Equation (4) and find the potential $\Phi$, we have to set the value of $r_{h 0}$. We define the heliosheath thickness $(\Delta r)$ in the $\theta=0^{\circ}$ direction as the difference $r_{h 0}-r_{s 0}$. Exarhos \& Moussas (2000) considered the value $\Delta r=37.6 \mathrm{AU}$ of the one-dimensional model of Khabibrakhmanov et al. (1996). For the sake of the first example in this study, we use the same $\Delta r$ value and we examine the Laplacian flow components on the shock. In Figure 2, we show the termination shock distance and the radial velocity component on the termination shock, $u_{r}\left(r=r_{s}\right)$, as a function of the latitude $\theta$. For $\theta=0^{\circ}$ the downstream speed $u_{s}$ is four times smaller than the upstream speed because of condition (11) but for angles $\theta>\sim 60^{\circ}$ the downstream flow is directed toward the upstream region, which is inconsistent with the model of the termination shock and the heliosheath flow. We note that for a range of small $\Delta r$ values, according to Equation (14), constant $\Delta$ is a large positive number resulting to negative $B_{0}$ values as implied from Equation (13). Then, according to Equation (7), as $\theta$ increases, the negative terms of the flow become the dominant terms, which lead to the inconsistency we just presented. 

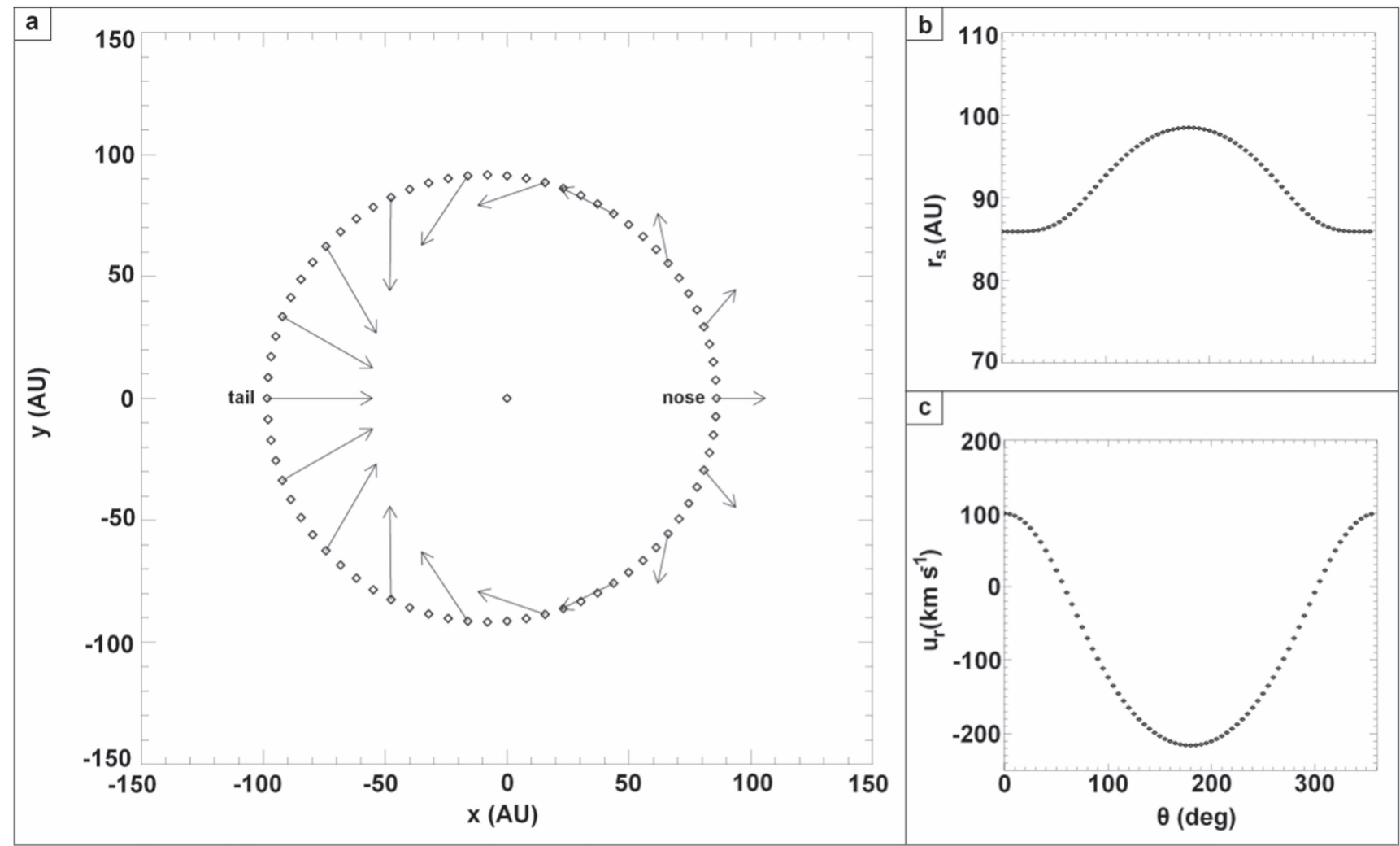

Figure 2. (a) The termination shock location for compression ratio $R=4$ and $\Delta r=37.6$ AU. The arrows represent the plasma flow vector on the downstream region of the shock. (b) The heliocentric distance of the termination shock and (c) The radial component of the downstream plasma velocity on the termination shock location as a function of $\theta$ angle. The mathematical model predicts polar and caudal elongation of the shock, but for non-physical heliosheath flows.

The specific model can lead to inconsistencies even for quite large $\Delta r$ and this can be seen if we carefully examine the equations. If the compression ratio $R$ does not change with the $\theta$ angle, then the downstream speed for $\theta=0^{\circ}$ and $\theta=180^{\circ}$ for example, according to 2(c) is $u_{s 0}=u_{s 180}=u_{1} / R$. Then, according to Equation (3) the termination shock heliocentric distance is the same for $\theta=0^{\circ}$ and for $\theta=180^{\circ}$, thus, the termination shock caudal elongation (nose to tail asymmetry) is inconsistent with the equations above. In addition, note that for the same radial distance, the condition $u_{s 0}=u_{s 180}=u_{1} / R$ is not consistent with Equation (7). It is, however, possible to get a consistent model if we keep more terms of the Laplacian potential in Equation (6) or solve for compressible and rotational downstream flow, but this would extremely complicate the analytical solution. Instead, we would like to examine an alternative modification that, under some constraints, would result in a physical representation of the heliosheath flow using the simplified flow potential of Equation (6).

\section{2. $\alpha_{1}$ and $\alpha_{s}$ Angles}

In order to have a consistent model, we need to guarantee that, besides the speed magnitude, angles $\alpha_{1}$ and $\alpha_{s}$ are consistent with the Rankine-Hugoniot conditions at the termination shock for all the flow lines. If we combine Equations 2(b), 2(c), we find

$$
\cos \alpha_{s}=\sqrt{\frac{\left(\frac{u_{1}}{u_{s}}\right)^{2}-1}{R^{2}-1}} \text { or } \alpha_{s}=\cos ^{-1}\left[\sqrt{\frac{\left(\frac{u_{1}}{u_{s}}\right)^{2}-1}{R^{2}-1}}\right] \text {, }
$$

and using Equation 2(c), we solve for the angle $\alpha_{1}$,

$$
\begin{gathered}
\cos \alpha_{1}=R \frac{u_{s}}{u_{1}} \cos \alpha_{s}=\sqrt{\frac{1-\left(\frac{u_{s}}{u_{1}}\right)^{2}}{1-R^{-2}}} \\
\text { or } \alpha_{1}=\cos ^{-1}\left[\sqrt{\frac{1-\left(\frac{u_{s}}{u_{1}}\right)^{2}}{1-R^{-2}}}\right] .
\end{gathered}
$$

According to Equation 2(b), we have $\alpha_{s}>\alpha_{1}$. The geometry shown in Figure 1 implies that

$$
\alpha_{s}-\alpha_{1}=\varphi=\tan ^{-1}\left(\frac{u_{s \theta}}{u_{s r}}\right) .
$$

Next, we will introduce a shock compression ratio that varies as a function of $\theta$ angle and resolves the consistency of the model as we keep the same order terms for the heliosheath flow. Note that, in this case, the Laplacian flow in the heliosheath contains only $R=R(\theta=0)$, while in the RankineHugoniot conditions $R$ is treated as an angular dependent $R(\theta)$, for each streamline separately. We develop an algorithm that tests a wide range of compression ratio values $R$ for each streamline and determines the specific compression ratio value for which Equations (3) and (18) are satisfied. Initially, we run the algorithm by performing a rather coarse scan through a wide range of compression ratio test values $(\sim 1<R<100)$ in order to get a first order estimation of $R$ as a function of $\theta$. We then perform a second run by narrowing down the $R$ test value 


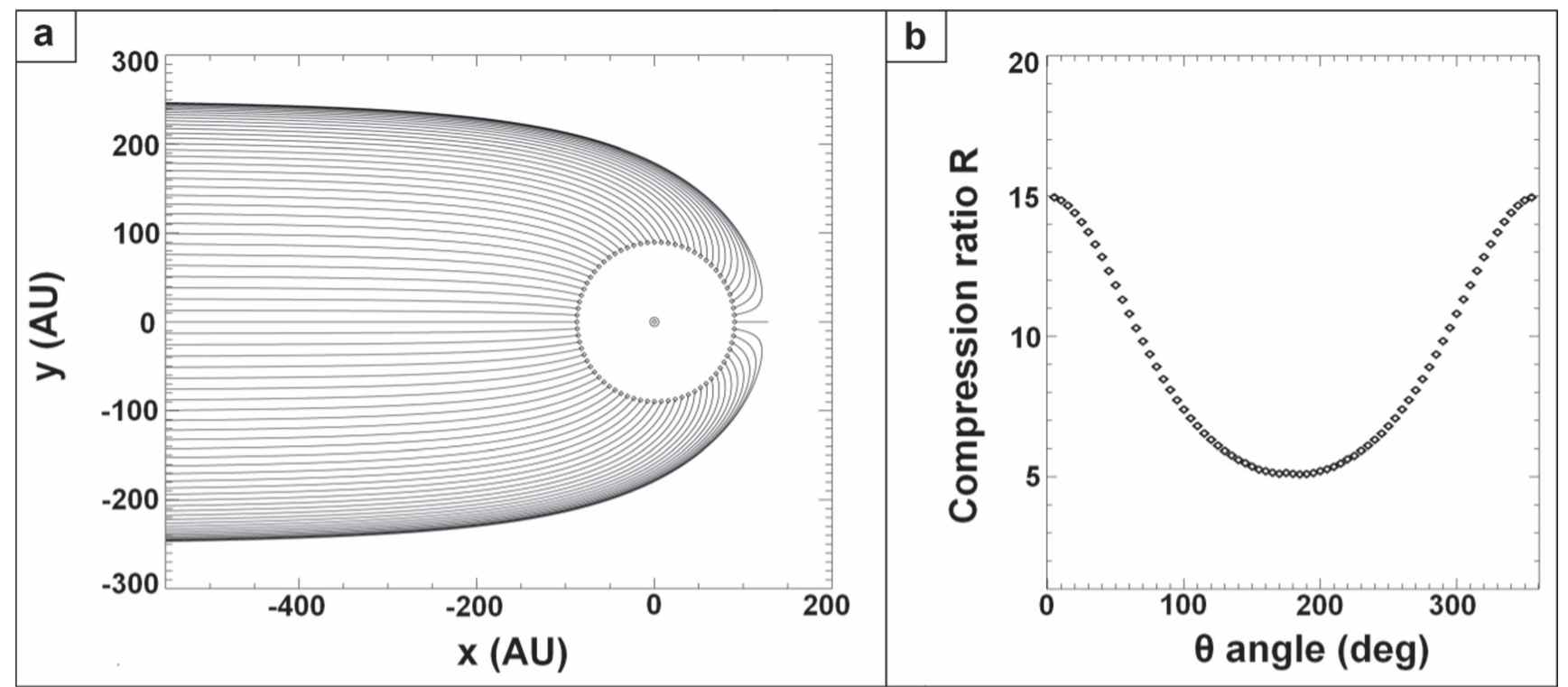

Figure 3. (a) The termination shock and the flow lines in the heliosheath assuming a varying shock compression ratio as a function of $\theta$ angle. The $r_{h}\left(\theta=0^{\circ}\right)-$ $r_{s}\left(\theta=0^{\circ}\right)$ length is set to $37.6 \mathrm{AU}$ and the compression ratio for $\theta=0^{\circ}$ is set to $R\left(\theta=0^{\circ}\right)=15$. (b) The compression ratio of the termination shock as a function of $\theta$.

range and increasing its precision. We repeat the runs until we reach the desired precision. We note that the $\alpha_{1}$ angle is depended on the termination shock geometry. For simplicity, we restrict our model to find solutions for small $\alpha_{1}$ angles $\left(<5^{\circ}\right)$ so that $\alpha_{s} \approx \varphi$. In order to solve for nearly spherical shock, we seek solutions where the radial distance of the shock for any $\theta$ does not differ more than $5 \%$ from the shock distance in the upwind direction $r_{\mathrm{s} 0}$.

\section{The Modified Model}

We first use the model with the modifications discussed in the previous section to investigate for what shock compression ratio profile we derive a physical and consistent heliosheath flow for $\Delta_{r}=r_{h}\left(\theta=0^{\circ}\right)-r_{s}\left(\theta=0^{\circ}\right)=37.6 \mathrm{AU}$. We use the upstream and interstellar parameters as mentioned above. We found that there are no acceptable solutions for the potential $\Phi$ if the compression ratio at $\theta=0^{\circ}, R\left(\theta=0^{\circ}\right)$ is less than $\sim 12$. In Figure 3(a), we present a self-consistent model of the termination shock and the heliosheath flow for the case where $R\left(\theta=0^{\circ}\right) \sim 15$, while in Figure 3(b), we show the termination shock compression ratio as a function of the $\theta$ angle for the specific example. The compression ratio along the termination shock decreases significantly toward the tail. For this example, the compression ratio is $\sim 5$ in the tail, which is $\sim 3$ times lower than the compression ratio at the shock's nose.

We then examine the shock location and shape, the heliosheath flow characteristics and the minimum compression ratio as a function of $\theta$ that is required to have physical solutions for several $\Delta r$ values. Figure 4 illustrates our results. We show that the minimum compression ratio, required for extracting reasonable streamlines, is larger for smaller $\Delta r$ values. For all the illustrated examples, the compression ratio decreases toward the tail. The variation of the compression ratio as a function of $\theta$ is also dependent on $\Delta r$. (Note that we kept only those solutions for which angle $\alpha_{1}$ is small and the shock is nearly spherical. For more complicated geometries, the function of the minimum compression ratio as a function of $\theta$ may be different.)

\section{Discussion and Conclusions}

We used a previously developed semi-analytical model for the termination shock location, and we examined the heliosheath flow for several boundary conditions. We showed the inconsistency of the model by calculating the angles between the flow and the front shock normal and comparing with the Rankine-Hugoniot conditions on the shock. We suggested a modified model that assumes a varying shock compression ratio along the termination shock, so the model can be self-consistent. The concept of this paper was to model and preserve consistent flow streamlines by considering incompressible and irrotational flow and solving accordingly the velocity field $\Phi$ and a semi-variable compression ratio along the termination shock. The variability of the Bernoulli constant in the entire flow must be of the order of the variability of the divergence of the velocity field, a condition that can be rigorous depending on the other parameter values. A future work may carry out specific patterns of the compression ratio angular dependence that can selectively be optimized in accordance with the Bernoulli constant and/or other physical constraints.

\subsection{Compression Ratio Profile}

We used the modified model to examine a case, where the heliosheath thickness toward the nose is $\Delta r=37.6$ AU (taken from reference), while the variable compression ratio is characterized by an angular, nearly harmonic, variation from $R \sim 15$ (for $\theta=0^{\circ}$ ) to $R \sim 5$ (for $\theta=180^{\circ}$ ), as shown in Figure 3(b). This corresponds to a polytropic index variation from $\gamma \sim 1.15$ (for $\theta=0^{\circ}$ ) to $\gamma \sim 1.5$ (for $\theta=180^{\circ}$ ), that is, on average $\gamma \sim 1.3$, corresponding to sub-adiabatic polytropic processes, as shown in Figure 5.

We further examine several other cases for different $\Delta r$ values and we presented the minimum compression ratio as a function of polar angle $\theta$ for each case. We specifically showed that (1) the minimum shock compression ratio increases for smaller heliosheath standoff thickness $\Delta r$, (2) the variation of the compression ratio as a function of the latitude $(\theta)$ is more 


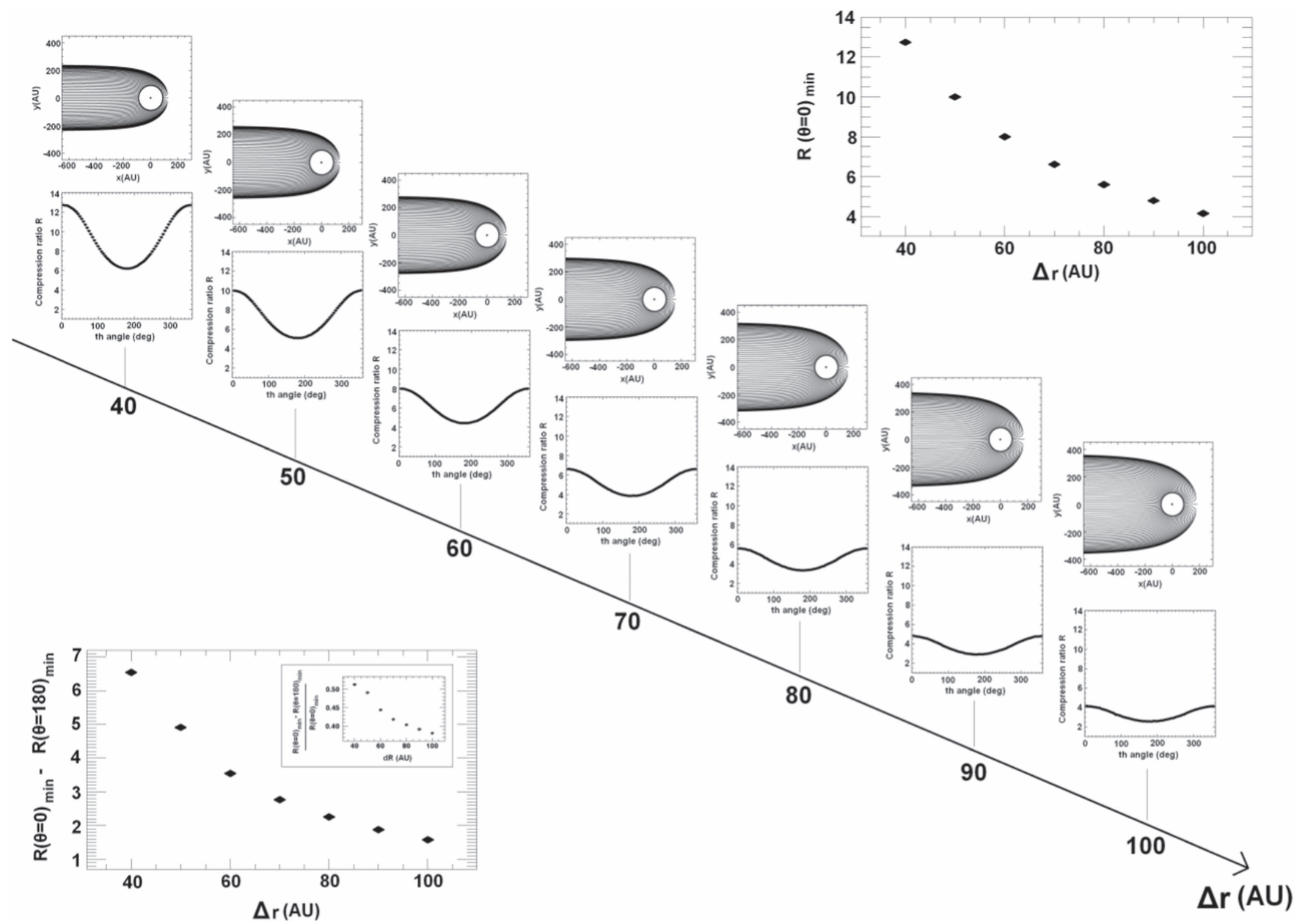

Figure 4. Heliosheath flow streamlines and the shock compression ratio are shown as functions of the polar angle $\theta$ and for several values of the inner heliosheath thickness, $\Delta r=r_{h}\left(\theta=0^{\circ}\right)-r_{s}\left(\theta=0^{\circ}\right)$. In the top right corner, we present the minimum consistent compression ratio for the shock nose and in the bottom left corner, we show the difference between the nose and tail compression ratio at the termination shock (the input panel plots the normalized difference).

pronounced for smaller $\Delta r$, and (3) for nearly adiabatic strong shock $(\gamma \sim 5 / 3, R \sim 4)$ the heliosheath standoff thickness $\Delta r$ is $\sim 100 \mathrm{AU}$.

\subsection{Downstream Flow Assumptions}

Parker (1961) demonstrated that the downstream flow is approximately incompressible. Combining the shock jump conditions for a nearly spherical, strong shock, the Bernoulli's integral, and the polytropic equation, we can express the downstream density along each streamline as

$$
\frac{\rho_{s}}{\rho_{i}}=\left[1+\frac{(\gamma-1)^{2}}{4 \gamma}\right]^{-\frac{1}{\gamma-1}} .
$$

where $\rho_{i}$ is the stagnation density. The expression above is valid for the cases where the polytropic index $\gamma$ is the same for the upstream solar wind and the heliosheath so that the shock compression ratio can be given by (2e). In the examples given in this study, the compression ratio varies from $\sim 3(\gamma=2)$ to $\sim 15 \quad(\gamma=8 / 7)$. For those examples, according to Equation (19), the plasma density in the heliosheath does not vary more than $\sim 12 \%$; thus, the incompressible assumption is not significantly off. The incompressibility assumption is weaker for lower compression ratios (higher $\gamma$ ).
In this study, we seek solutions where the shock is nearly spherical around the Sun. We still allowed for small $\alpha_{1}$ angles, which can result to non-negligible $\alpha_{s}$ angles when the compression ratio is high. The small deviations from nonelongated shock that resulted from our simplified approach could be explained by the fact that the heliosheath flow is not strictly irrotational (for details, see Parker 1961; Suess \& Nerney 1990).

\subsection{Shock Strength}

In this study, we investigated the termination shock under the assumption of a strong, nearly perpendicular shock. We have already discussed how more complexity can be added by changing the geometry of the shock and still guarantee a selfconsistent model. Even more complexity can be added when the strong shock assumption is dropped. In this case, the upstream Mach number is not infinity and the upstream plasma temperature should be included in our calculations. The termination shock is expected to be weak due to solar wind deceleration and heat from pick-up ions. Richardson et al. (2008) studied the first crossings of the termination shock by Voyager 1 and 2. The shock compression ratio was found to be around 2, indicating a low Mach number plasma in the case of adiabatic plasma $(\gamma=5 / 3)$. For this case, the RankineHugoniot equations need to be modified to include the 


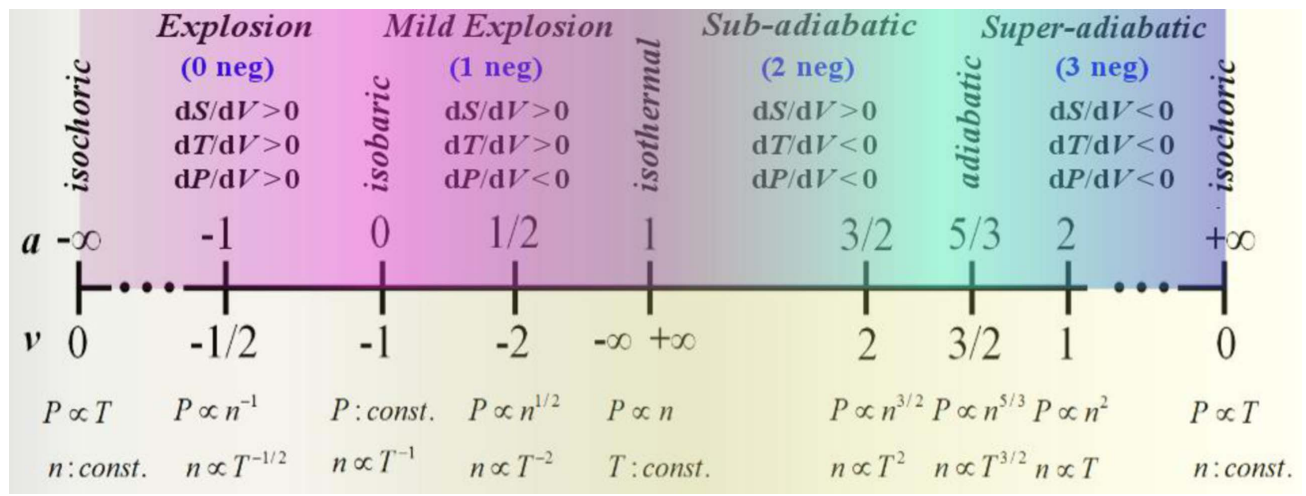

Figure 5. Polytropic spectrum: arrangement of thermodynamic processes along the interval of the (constant) polytropic index $a$ or $\nu \equiv 1 /(a-1)$. Starting from $a \rightarrow-\infty$ (isochoric) and moving with increasing $a$ (left-to-right), there are four intervals of "Explosion," "Mild Explosion," "Sub-adiabatic," and "Super-adiabatic," which are characterized by numbers of $0,1,2$, and 3 negative terms (noted in blue) out of the three $\{d S / d V, d T / d V, d P / d V\}$, as explained by Livadiotis (2016). The examined model considers a polytropic index between isothermal and adiabatic processes, that is, in the sub-adiabatic region. (Taken from Livadiotis 2016.)

momentum and energy terms that are related to the pick-up process (e.g., see Li et al. 2008). Some fluid and MHD models study the termination shock and the heliosheath under the weak shock approach (Zank 1999, and references therein). In our study, instead, we keep the strong shock approach in order to exhibit the inconsistency that can arrive even from a previously developed, simplified semi-analytical model. We have shown how the model becomes self-consistent (not necessarily consistent with real case), while we keep the strong shock approach and vary the polytropic index of the plasma to achieve the compression ratio variations with latitude. Under those simplifications, the nearly adiabatic plasma case will constrain the heliosheath thickness to $\Delta r \approx 100 \mathrm{AU}$.

\section{References}

Baranov, V. B., \& Malama, Y. G. 1993, JGR, 98, 15157

Barnes, A. 1998, JGR, 103, 2015

Exarhos, G., \& Moussas, X. D. 1999a, SoPh, 187, 145

Exarhos, G., \& Moussas, X. D. 1999b, SoPh, 187, 157

Exarhos, G., \& Moussas, X. D. 2000, ApJ, 542, 1075

Hugoniot, P. H. 1887, Mémoire sur la propagation du mouvement dans les corps et ples spécialement dans les gaz parfaits, 1e Partie, 57 (Paris: J. Ecole Polytech)
Hugoniot, P. H. 1889, Mémoire sur la propagation du mouvement dans les corps et plus spécialement dans les gaz parfaits, 2e Partie, 58 (Paris: J. Ecole Polytech)

Khabibrakhmanov, I. K., Summers, D., Zank, G. P., \& Pauls, H. L. 1996, JGR, 101, 20003

Li, H., Wang, C., \& Richardson, J. D. 2008, GeoRL, 35, L19107

Lipatov, A. S., Zank, G. P., \& Pauls, H. L. 1998, JGR, 103, 20631

Livadiotis, G. 2015, ApJ, 809, 111

Livadiotis, G. 2016, ApJS, 223, 13

Livadiotis, G., \& Desai, M. I. 2016, ApJ, 829, 88

Livadiotis, G., \& McComas, D. J. 2012, ApJ, 749, 11

Livadiotis, G., \& McComas, D. J. 2013, JGRA, 118, 2863

Nerney, S., \& Suess, S. T. 1995, GeoRL, 22, 1757

Nicolaou, G., Livadiotis, G., \& Moussas, X. 2014, SoPh, 289, 1371

Parker, E. N. 1958, ApJ, 128, 664

Parker, E. N. 1961, ApJ, 134, 20

Pauls, H. L., \& Zank, G. P. 1996, JGR, 101, 17081

Pauls, H. L., Zank, G. P., \& Williams, L. L. 1995, JGR, 100, 21595

Pogorelov, N. V. 2016, J. Phys.: Conf. Ser., 719, 012013

Rankine, W. J. M. 1870, RSPTA, 160, 277

Richardson, J. D., Kasper, J. C., Wang, C., Belcher, J. W., \& Lazarus, A. J. 2008, Natur, 454, 63

Senanayake, U. K., \& Florinski, V. 2013, ApJ, 778, 122

Steinolfson, R. S. 1994, JGR, 99, 13307

Steinolfson, R. S., \& Gurnett, D. A. 1995, GeoRL, 22, 651

Steinolfson, R. S., Pizzo, V. J., \& Holzer, T. 1994, GeoRL, 21, 245

Suess, S. T., \& Nerney, S. 1990, JGR, 95, 6403

Wang, C., \& Belcher, J. W. 1998, JGR, 103, 247

Zank, G. P. 1999, SSRv, 89, 413 